\title{
What activates visceral afferents?
}

\author{
D Grundy
}

Gut 2004;53(Suppl II):ii5-ii8. doi: 10.1136/gut.2003.033415

Vagal and spinal afferents represent the information superhighways that convey sensory information from the gut to the central nervous system. These afferents are sensitive to both mechanical and chemical stimuli. Vagal afferents terminate in the muscle layers and in the mucosa. Muscle afferents are activated at physiological levels of distension and during peristalsis. In contrast, spinal afferents encode supraphysiological levels of intestinal pressure. Vagal and spinal afferents also express a wide range of membrane receptors to a variety of chemical mediators generated from both within and outside the gut wall. Some of these receptors are part of a modality specific transduction pathway involved in sensory signalling from the gut lumen to vagal afferent endings in the mucosa. Others, which are activated by substances derived from multiple cellular sources during ischaemia, injury, or inflammation act in a synergistic way to cause acute or chronic sensitisation of the afferent nerves to mechanical and chemical stimuli. Understanding the mechanisms that underlie hypersensitivity may have implications for the pharmaceutical approach to the treatment of functional bowel disorders like irritable bowel syndrome.

Correspondence to:

D Grundy, Department of Biomedical Science, University of Sheffield

Western Bank, Sheffield S10 2TN, UK; d.grundy@ sheffield.ac.uk

Accepted for publication 16 October 2003
$\mathrm{T}$ he mechanisms involved in visceral sensation are generally less well understood than their corresponding sensations in the somatic realm but there are a number of features in common. Similar types of nerve-for example, small diameter unmyelinated (C) or thinly myelinated $(\mathrm{A} \delta)$ fibres-convey both visceral and somatic painful sensations. Visceral pain is poorly localised and is often referred to somatic sites because the spinal afferent fibres converge in the dorsal horn of the spinal cord. However, the thoracic and abdominal viscera also project sensory information to the brainstem via vagal afferent pathways. These two classes of visceral extrinsic nerves (vagal and spinal) exhibit contrasting stimulus response properties, which in turn, reflects their diverse roles in sensory signalling.

\section{MECHANOSENSITIVITY} afferents can be localised within the GI wall using immunocytochemistry and other fibre tracing techniques. Their location in mucosal layers, muscle, and in the serosal and mesenteric attachments are consistent with their responses
The peripheral terminals of vagal and spinal to stimuli acting at these different sites within the GI wall. ${ }^{1}$ Nerve terminals in the serosa and in the muscle convey mechanosensory information relevant to distension and contraction of the bowel wall. However, the afferent information generated from these sites and conveyed by spinal and vagal mechanosensitive afferents respectively is very different, as revealed by direct electrophysiological recordings of afferent traffic on route to the $\mathrm{CNS}$. $^{2}$

Vagal muscle mechanoreceptors have low thresholds of activation and reach maximal responses within physiological levels of distension (fig 1). In contrast, many spinal afferents with endings in the serosa and mesenteric attachments are able to respond beyond the physiological range and encode both physiological and noxious levels of stimulation. This different stimulus-response profile is consistent with the hypothesis that vagal afferents are involved in physiological regulation while spinal afferents are responsible for mediating pain.

\section{VAGAL MECHANORECEPTORS}

Two types of vagal ending, IMAs and IGLEs, have been attributed to mechanosensory function in the gut. ${ }^{3}{ }^{4}$ IMAs have been suggested to be "in-series tension receptor endings" that respond to muscle tension generated during passive stretch or active contraction of the muscle. However, more recently this mechanosensory property has been attributed to IGLEs, which are found as basket like structures surrounding myenteric ganglia. ${ }^{4}$ Evidence supporting this view has been elaborated recently by mapping the receptor fields of vagal afferent endings in the oesophagus and showing morphologically that these "hot spots" correspond to the location of IGLEs. ${ }^{5}$ This mechanosensitivity is suggested to arise because IGLEs are associated with connective tissue elements that would be exposed to shearing forces during stretch muscle contraction. However, another intriguing possibility arising from the close proximity of IGLEs to the

Abbreviations: $\mathrm{Gl}$, gastrointestinal; $\mathrm{CNS}$, central nervous system; IMAs, intramuscular arrays; IGLEs,

intraganglionic laminar endings; $E C$, enterochromaffin; 5-HT, 5-hydroxytryptamine; CCK, cholecystokinin; PLC, phospholipase C; PGs, prostaglandins; ATP adenosine triphosphate, ; NSCCs, non-selective cation channels; cAMP, cyclic adenosine 3', 5'-monophosphate; PLA2, phospholipase A2; AC, adenylate cyclase; PARs, protease activated receptors; COX-1, COX-2, cyclooxygenase-1 and 2; AA, arachidonic acid; DAG, diacylglycerol; IP3, inositol 1,4,5-trisphosphate 
A

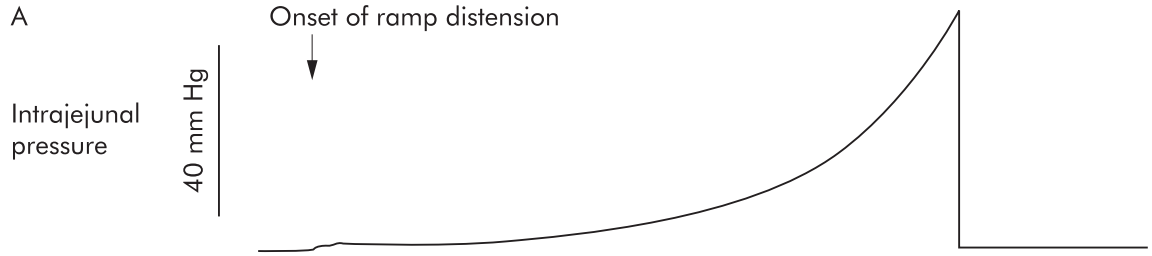

B

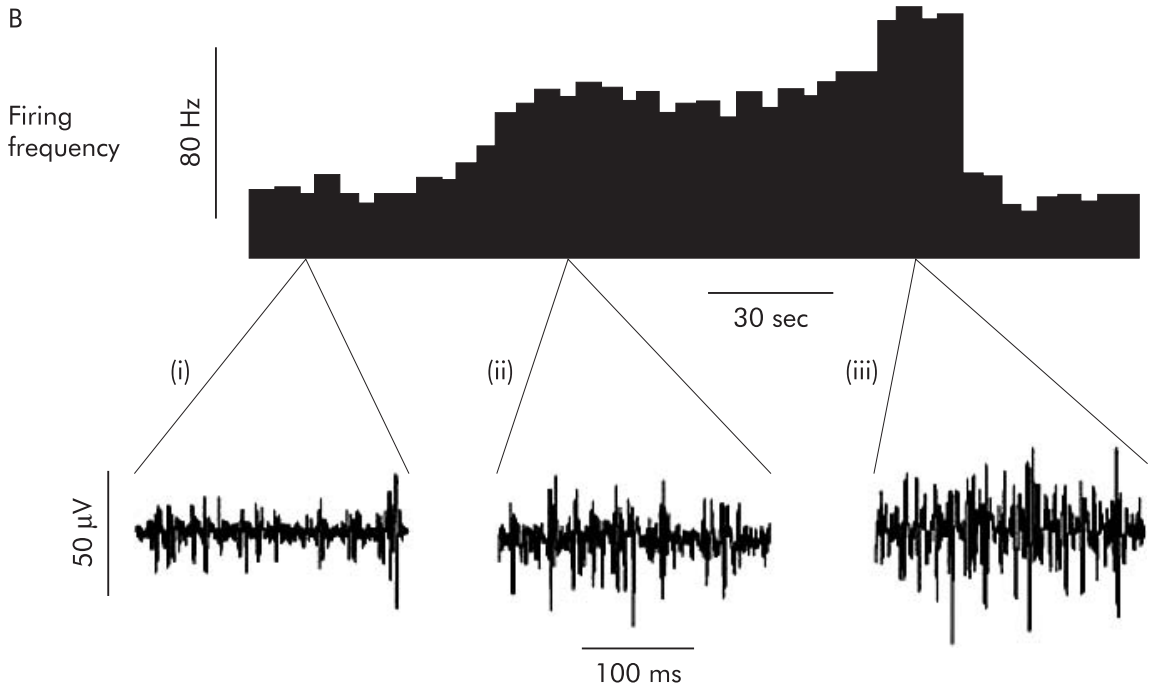

Figure 1 Whole nerve mesenteric afferent recording from the rat jejunum showing a biphasic pattern of mechanosensitivity during ramp distension to $60 \mathrm{mmHg}$. The top trace is intrajejunal pressure, the middle trace shows a sequential rate histogram of afferent firing frequency, and below that are snapshots of the neurogram taken at baseline and during the low and high threshold phases of the response to distension. Note the prominent increase in firing at low levels of distension is a reflection of low threshold mechanosensitive afferents, whose response plateaus until intrajejunal pressure reaches threshold for activation of high threshold afferents. Reprinted from Booth et al (Gastroenterology 2001;121:358-69) with permission from the American Gastroenterological Association. myenteric ganglion is that IGLEs are chemosensitive, responding to neurotransmitters and neuromodulators released into the synaptic neuropil. ${ }^{6}$ In the absence of clearly defined synapses between IGLEs and myenteric neurons, communication may arise following simple diffusion from the site of release to the afferent nerve terminals. Many cell types (for example, neurons, glial cells, endothelium) and many different kinds of substances (ions, purines, amino acids, monoamines, peptides, gases) released through vesicular or non-vesicular mechanisms could potentially participate in such communication.

\section{SPINAL MECHANORECEPTORS}

Spinal afferents respond to distension over a wide dynamic range extending from the physiological to noxious levels. ${ }^{7}$ These spinal endings can contribute to signalling visceral pain through some intensity code that recognises extreme levels of distension or contraction. Other spinal afferents, however, respond only to noxious levels of distension and an extreme example of these is the high threshold mechanoreceptor that fails to respond under normal circumstances. These are the so called "sleeping" or silent nociceptors that can be awakened under conditions of injury or inflammation. ${ }^{8}$ The latter illustrate the fact that mechanosensitivity is not fixed either in terms of threshold for activation or gain in the stimulus-response relation and, as such, the threshold can be reduced and the gain increased under certain situations.

\section{CHEMOSENSITIVITY}

There is an enormous range of chemical mediators that influence the sensitivity of visceral afferents. Electrophysiological, immunocytochemical, and molecular biological techniques have revealed the functional expression of receptors to various mediators on the cell bodies of visceral sensory neurons in the dorsal root or nodose ganglia or on their processes in the gut wall. These mediators produce their effects on visceral afferent nerves by three distinct processes. Firstly, by direct activation, which generally involves the opening of ion channels present on the nerve terminals. Secondly, by sensitisation, which may occur in the absence of a direct stimulation, but which usually results in afferent hyperexcitability to both chemical and mechanical modalities. Thirdly, by altering the phenotype of the afferent nerve, for example through alterations in the expression of mediators, channels, and receptors or modulating the activity of these by changing the ligand binding characteristics or coupling efficiency of other receptors.

\section{SENSORY SIGNAL TRANSDUCTION}

Mediators that produce a direct stimulation of visceral sensory nerve endings may do so as part of a discrete sensory signalling pathway. In this case, the afferent neuron does not respond directly to a stimulus, but following the release of a mediator from a primary sense cell. Examples of these cells, which effectively act as principal sensory transducers, are enterochromaffin (EC) cells, which release 5-hydroxytryptamine (5-HT) and enteroendocrine cells that release CCK. These epithelial cells are strategically positioned in the intestinal mucosa to "taste" luminal contents and release their mediators across the basolateral membrane in order to generate action potentials in the afferent nerve endings within the lamina propria." Electrophysiological evidence suggests that 5-HT and CCK act on distinct subpopulations of vagal mucosal afferent nerves. ${ }^{10}$ As such, this sensitivity represents an example of a high fidelity, modality specific signal transduction pathway. This mechanism, more than 


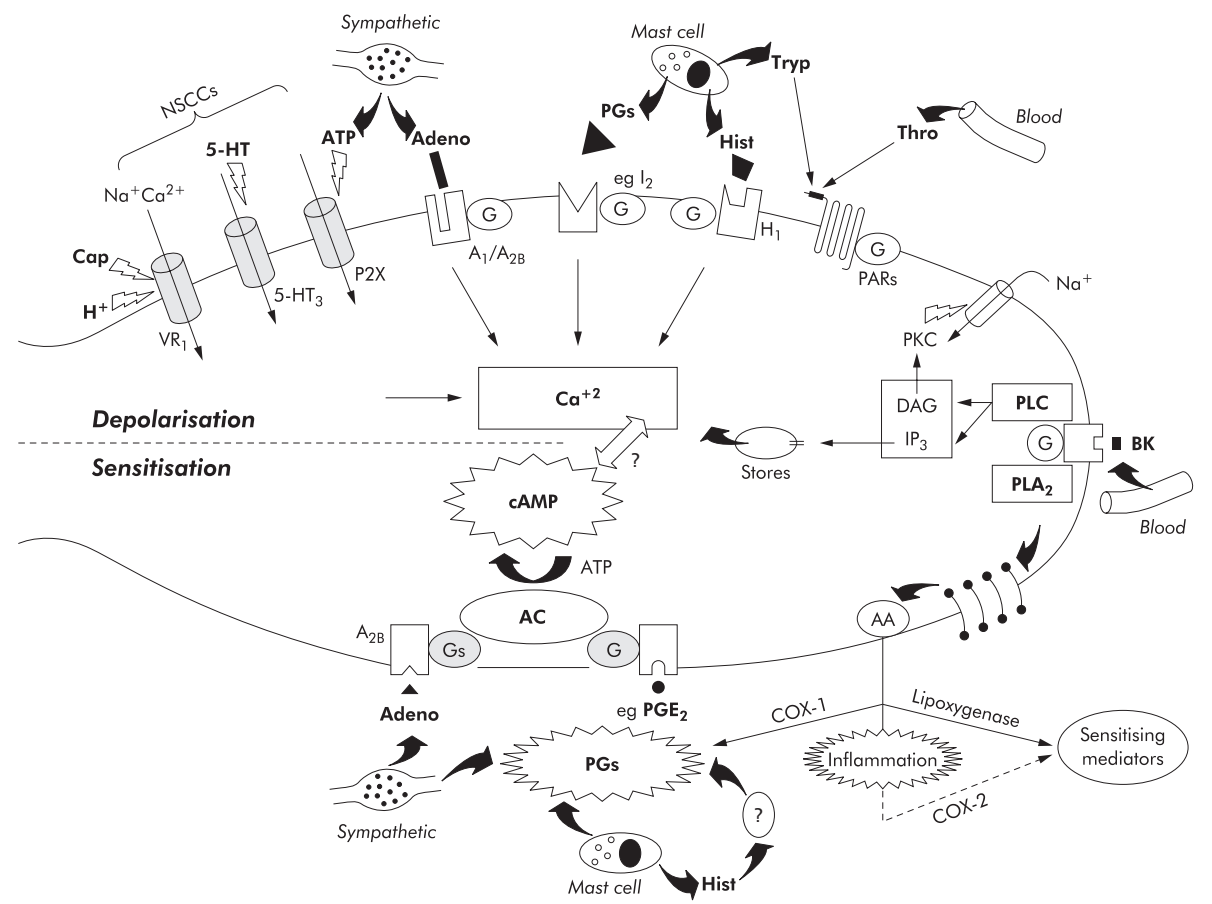

Figure 2 Some of the potential receptor mechanisms underlying activation and sensitisation of gastrointestinal sensory afferents. Mediators such as serotonin (5-HT) cause activation whereas others, like PGE2, sensitise visceral afferent responses to other stimuli. Others, for example adenosine (Adeno), cause both stimulation and sensitisation, possibly through distinct receptor mechanisms. Bradykinin has a self sensitising action, stimulating discharge through activation of phospholipase C (PLC) and enhancing excitability via prostaglandins (PGs) following activation of phospholipase A2 (PLA2). Inflammatory mediators can be released from different cell types (such as sympathetic nerves, mast cells, and blood vessels) present in or around the afferent nerve terminal. 5-HT, adenosine triphosphate (ATP) and capsaicin (Cap) can directly activate non-selective cation channels (NSCCs) while adenosine, histamine, prostaglandins (not PGE2), and proteases such as mast cell tryptase (Tryp) and thrombin (Thro) act on G-protein coupled receptors leading to a $\mathrm{Ca} 2+$ dependent modulation of ion channel activity. Sensitisation, however, may be mediated by raised intracellular cyclic adenosine $3^{\prime}, 5^{\prime}$-monophosphate (cAMP). Adenosine and PGE2 can generate cAMP directly through G-protein-coupled stimulation of adenylate cyclase (AC). In contrast, histamine may act indirectly through the generation of prostaglandins (8). The actions of cAMP downstream may involve modulation of ion channels, interaction with other second messengers (eg $\mathrm{Ca} 2+$ ), or even changes in receptor expression. Other abbreviations: protease activated receptors (PARs), cyclooxygenase-1 and 2 (COX-1, COX-2), arachidonic acid (AA), diacylglycerol (DAG), inositol 1,4,5trisphosphate (IP3). Reprinted from Kirkup et al (Am J Physiol Gastrointest Liver Physiol 2001;280:G787-94) with permission from the American Physiological Society.

likely, functions in the detection of moment to moment changes in luminal composition and operates, in the main, below the level of consciousness.

\section{PROMISCUOUS CHEMOSENSITIVITY}

In contrast to the specific signalling pathways that exist in vagal mucosal afferents, it is apparent that a battery of mediators can influence the sensitivity of spinal afferents in a more promiscuous manner. Such substances are usually released under conditions of inflammation, injury, or ischaemia from a plethora of cell types-for example, platelets, leukocytes, lymphocytes, macrophage, mast cells, glia, fibroblasts, blood vessels, muscle, and neurons. Each of these specific cells (for example, mast cells) may release several of these modulating agents, some of which may act directly on the sensory nerve terminal while others may act indirectly, following release of other agents from other cells in a series of cascades. Some of the key mediators, their cellular source and their action on visceral afferents are illustrated in figure 2 .

\section{CONCLUSIONS AND SUMMARY}

Afferent fibres convey a vast amount of sensory information to the brainstem and spinal cord, but the nature of this information is different for vagal and spinal pathways.
Vagal afferents convey predominantly physiological information, whereas spinal afferents are able to encode noxious events. These spinal nociceptors are influenced by peripherally acting chemicals, released during inflammation and injury, which are thought to trigger the processes leading to sensitisation and increased nociceptive activity. Other chemicals act in a more selective way to activate vagal afferents and are implicated in nutrient signalling from the GI tract.

\section{REFERENCES}

1 Berthoud B, Neuhuber WL. Functional and chemical anatomy of the afferent vagal system. Auton Neuroscience 2000;85:1-17.

2 Booth CE, Kirkup AJ, Hicks GA, et al. Somatostatin sst2-receptor mediated inhibition of mesenteric afferent nerves supplying the rat jejunum. Gastroenterology 2001;121:358-69.

3 Berthoud JR, Powley TL. Vagal afferent innervation of the rat fundic stomach: morphological characterization of the gastric tension receptor. J Comp Neurol 1992;319:261-76.

4 Phillips RJ, Powley TL. Tension and stretch receptors in gastrointestinal smooth muscle: re-evaluating vagal mechanoreceptor electrophysiology. Brain Res Rev 2000;34:1-26.

5 Zagorodnyuk VP, Brookes SJ. Transduction sites of vagal mechanoreceptors in the guinea pig esophagus. J Neurosci 2000; 20:6249-55.

6 Jiang W, Kirkup AJ, Kreis ME, et al. Nicotinic activation of vagal afferent fibres supplying the rat jejunum: "cross-talk" between the enteric and extrinsic innervation? Gastroenterology 2001; 120:A-1178. 
7 Gebhart GF. Pathobiology of visceral pain: molecular mechanisms and therapeutic implications IV. Visceral afferent contributions the pathobiology of visceral pain. Am J Physiol

2000; 278:G834-8.

8 Kirkup AJ, Brunsden AM, Grundy D. Receptors and transmission in the brain-gut axis: potential for novel therapies. Receptors on visceral afferents. Am J Physiol Gastrointest Liver Physiol 2001;280:G787-94.

9 Raybould HE. Nutrient tasting and signaling mechanisms in the gut. Sensing of lipid by the intestinal mucosa. Am J Physiol 1999;277:G751-5.

10 Hillsley K, Grundy D. Serotonin and cholecystokinin activate different populations of rat mesenteric vagal afferents. Neurosci Lett 1998;255:63-6. 